\title{
Uniform continuity and quantization on bounded symmetric domains
}

Article

Accepted Version

Bauer, W., Hagger, R. and Vasilevski, N. (2017) Uniform continuity and quantization on bounded symmetric domains. Journal of the London Mathematical Society, 96 (2). pp. 345366. ISSN 1469-7750 doi: https://doi.org/10.1112/jlms.12069 Available at https://centaur.reading.ac.uk/84022/

It is advisable to refer to the publisher's version if you intend to cite from the work. See Guidance on citing.

To link to this article DOI: http://dx.doi.org/10.1112/jlms.12069

Publisher: Wiley

All outputs in CentAUR are protected by Intellectual Property Rights law, including copyright law. Copyright and IPR is retained by the creators or other copyright holders. Terms and conditions for use of this material are defined in the End User Agreement.

\section{www.reading.ac.uk/centaur}

\section{CentAUR}

Central Archive at the University of Reading

Reading's research outputs online 


\title{
UNIFORM CONTINUITY AND QUANTIZATION ON BOUNDED SYMMETRIC DOMAINS
}

\author{
W. BAUER, R. HAGGER, AND N. VASILEVSKI
}

\begin{abstract}
We consider Toeplitz operators $T_{f}^{\lambda}$ with symbol $f$ acting on the standard weighted Bergman spaces over a bounded symmetric domain $\Omega \subset \mathbb{C}^{n}$. Here $\lambda>$ genus -1 is the weight parameter. The classical asymptotic relation for the semi-commutator

$$
\lim _{\lambda \rightarrow \infty}\left\|T_{f}^{\lambda} T_{g}^{\lambda}-T_{f g}^{\lambda}\right\|=0, \text { with } \quad f, g \in C\left(\overline{\mathbb{B}^{n}}\right),
$$

where $\Omega=\mathbb{B}^{n}$ denotes the complex unit ball, is extended to larger classes of bounded and unbounded operator symbol-functions and to more general domains. We deal with operator symbols that generically are neither continuous inside $\Omega$ (Section 4) nor admit a continuous extension to the boundary (Section 3 and 4 ). Let $\beta$ denote the Bergman metric distance function on $\Omega$. We prove that $(*)$ remains true for $f$ and $g$ in the space $\operatorname{UC}(\Omega)$ of all $\beta$-uniformly continuous functions on $\Omega$. Note that this space contains also unbounded functions. In case of the complex unit ball $\Omega=\mathbb{B}^{n} \subset \mathbb{C}^{n}$ we show that $(*)$ holds true for bounded symbols in $\operatorname{VMO}\left(\mathbb{B}^{n}\right)$, where the vanishing oscillation inside $\mathbb{B}^{n}$ is measured with respect to $\beta$. At the same time $(*)$ fails for generic bounded measurable symbols. We construct a corresponding counterexample using oscillating symbols that are continuous outside of a single point in $\Omega$.
\end{abstract}

\section{INTRODUCTION}

Let $\Omega \subset \mathbb{C}^{n}$ be a bounded symmetric domain (shortly BSD) and consider a (suitable) algebra of functions on $\Omega$. It is a classical scheme in deformation quantization to construct an associated family of non-commutative algebras $\mathcal{A}_{\lambda}$ that depend on a deformation parameter $\lambda$, and such that in the semi-classical limit (i.e. when the Planck constant $\hbar \sim \frac{1}{\lambda}$ tends to zero) $\mathcal{A}_{\lambda}$ should approach in some sense the above commutative algebra of functions, cf. [7, 6]. A classical method for constructing a deformation quantization of symmetric spaces uses Toeplitz operators as quantum counterparts of the functions we start with. Such operators are defined on the standard weighted Bergman spaces over $\Omega$, cf. [1, 11, 12, 15, 20, 22] and the weight parameter explicitly appears in the density function of the (Lebesgue) measure

Date: February 10, 2018.

2010 Mathematics Subject Classification. Primary: 47B35; Secondary: 81S10, 32M15 .

Key words and phrases. (mean) oscillation, Berezin transform, semi-commutator, semi-classical limit.

The first and third author acknowledge support through DFG (Deutsche Forschungsgemeinschaft), BA 3793/4-1. 
restricted to $\Omega$. Essential relations that one needs to prove (cf. [22]) are the norm convergence $(*)$ and (assuming some smoothness of the symbols) the second order asymptotic

$$
\left\|\left[T_{f}^{\lambda}, T_{g}^{\lambda}\right]-\frac{i}{\lambda} T_{\{f, g\}}^{\lambda}\right\|=O\left(\lambda^{-2}\right) \quad \text { as } \quad \lambda \rightarrow \infty .
$$

Here $[\cdot, \cdot]$ denotes the commutator of operators and $\{\cdot, \cdot\}$ is the Poisson bracket which is associated to a symplectic form induced by the Bergman metric tensor.

Recall that a quantization via Toeplitz operators acting on the Bergman space was first introduced by F. Berezin [6, 7, 8] for the case of the unit disk $\mathbb{D}$ in the complex plane and, more generally, for BSDs $\Omega \subset \mathbb{C}^{n}$. An a bit different approach to quantization for the unit disc $\mathbb{D}$ has been considered by Klimek and Lesniewski in [20] and subsequently was generalized to arbitrary BSDs by Borthwick, Lesniewski and Upmeier in [12]. Deformation estimates for Berezin-Toeplitz quantization on the Euclidean $n$-space $\Omega=\mathbb{C}^{n}$ equipped with a family of Gaussian measures were obtained in [11, 14]. In this non-compact setting the proofs are based on the relation between Toeplitz operators and pseudo-differential operators in Weyl-quantization. In particular, the required norm estimates are a consequence of the Calderon-Vaillancourt theorem. For $\Omega$ being a compact Kähler manifold, the above asymptotic relations have been obtained by Bordemann, Meinrenken and Schlichenmaier [10] (see also [21]). An analysis of the semi-classical limit for smoothly bounded strictly pseudoconvex domains in $\mathbb{C}^{n}$ can be found in [15]. We mention as well that a family of associative star products in deformation quantization can be constructed on the base of $(*)$ and $(* *)$, cf. [15].

The above mentioned results typically require certain regularity of the operator symbols and their controlled behavior close to the boundary of the domain (or at infinity). More precisely, in [12, Theorem 2.2] the relation $(*)$ is proved assuming that $f$ and $g$ are bounded continuous functions and $g$ has compact support in $\Omega$. In the special case of $\Omega=\mathbb{B}^{n}$ we may as well apply [15, Theorem 3], which assumes that $f$ and $g$ are smooth up to the boundary of $\Omega$. If one is only interested in $(*)$, this assumption can be relaxed to $f, g \in C(\bar{\Omega})$ by a simple approximation argument.

The aim of the present paper is to extend $(*)$ for symbols $f, g$ in larger algebras of bounded (and unbounded) functions in BSDs $\Omega$. We show that $(*)$ holds true if $f$ and $g$ are bounded and uniformly continuous in $\Omega$ with respect to the Bergman metric distance $\beta$. Note that in general such functions do not extend continuously to the boundary $\partial \Omega$. Moreover, we can even drop the boundedness assumption and obtain $(*)$ for (unbounded) Toeplitz operators with $\beta$-uniformly continuous symbols (cf. Theorem 3.8). At the same time Example 3.15 shows that $(*)$ may fail if we drop the continuity assumption even in one single point inside $\Omega$. In the last section of the paper we deal only with the complex unit ball $\Omega=\mathbb{B}^{n}$. We emphasize that a controlled oscillation of bounded symbols $f$ and $g$ inside $\Omega$ implies $(*)$. To be precise, assuming that $f$ or $g$ belongs to the space $\operatorname{VMO}\left(\mathbb{B}^{n}\right)$ of bounded functions having 
vanishing oscillation with respect to $\beta$ is sufficient for $(*)$. Our proofs use a refinement of the norm estimates for Hankel operators in [5] and an asymptotic analysis of the $\mathrm{BMO}^{\lambda}$-seminorms of $\beta$-uniformly continuous functions (cf. Proposition 3.4 and 3.7). We remark that by different methods similar (but slightly weaker) results for the Fock space case (i.e. $\Omega=\mathbb{C}^{n}$ equipped with a family of Gaussian measures) have been obtained recently in [1].

One of our motivations for considering this problem stems from the representation theory of $C^{*}$-algebras generated by Toeplitz operators (cf. [4]). In fact, in this paper a family of irreducible representations has been constructed under certain assumptions which include (*). We expect our analysis to be useful for the study of Toeplitz $C^{*}$-algebras with generating operators having symbols in (suitable) classes of functions that not necessarily admit continuous boundary values. Further details shall be presented in a forthcoming work.

In Section 2 we fix the notation and present some standard material on BSDs, Bergman spaces and Toeplitz operators. In particular, we show that finite products of (i.g. unbounded) Toeplitz operators with $\beta$-uniformly continuous symbols are well-defined on a common dense domain. We start Section 3 with some technical estimates and use them to derive a norm estimate for Hankel operators, which is needed in the proof of our main result (Theorem 3.8). The compactness of semi-commutators are discussed and we present the above mentioned counterexample. Finally, in Section 4 we prove $(*)$ in the case of bounded symbols having vanishing oscillation inside $\Omega=\mathbb{B}^{n}$.

\section{Preliminaries}

Throughout the paper we consider a BSD $\Omega \subset \mathbb{C}^{n}$ in its Harish-Chandra realization [5, 13, 15, 17, 24]. In particular, $\Omega$ contains the origin and is convex and circular. We write $G=\operatorname{Aut}_{0}(\Omega)$ for the connected component of the automorphism group of $\Omega$ which contains the identity. By $K$ we denote the (maximal) subgroup of $G$ that stabilizes the origin.

As is well-known, each $k \in K$ extends to a linear mapping on $\mathbb{C}^{n}$ [13]. If $r$ denotes the rank of $\Omega$, then there is a set $\left\{f_{1}, \cdots, f_{r}\right\} \subset \mathbb{C}^{n}$ (Jordan frame) of $\mathbb{R}$-linear independent vectors such that

$$
\Omega=\left\{z \in \mathbb{C}: z=k \sum_{j=1}^{r} t_{j} f_{j}, k \in K, 1>t_{1} \geq t_{2} \geq \cdots \geq t_{r} \geq 0\right\} .
$$

The sum-representation of $z \in \Omega$ in (2.1) is called polar decomposition and, assuming the above ordering, the numbers $t_{j}$ are uniquely determined ( $k$ is not i.g.). There is a polynomial (Jordan triple determinant)

$$
h: \mathbb{C}^{n} \times \mathbb{C}^{n} \rightarrow \mathbb{C}
$$

holomorphic in $z$ and anti-holomorphic in $w$ which restricted to the diagonal fulfills

$$
h(z, z)=\prod_{j=1}^{r}\left(1-t_{j}^{2}\right) .
$$


Moreover, $h$ is conjugate symmetric, i.e. $h(z, w)=\overline{h(w, z)}$ and invariant under the action of $K$, i.e. for all $z, w \in \Omega$ and all $k \in K$

$$
h(k z, k w)=h(z, w) .
$$

Let $p$ be the genus of $\Omega$ (see [15] for the definition) and $\lambda>p-1$. Consider the following weighted measure on $\Omega$ :

$$
d v_{\lambda}(z)=c_{\lambda} h(z, z)^{\lambda-p} d v(z),
$$

where $d v$ denotes the normed to one Lebesgue measure on $\Omega$ and $c_{\nu}>0$ is a normalizing constant such that $v_{\lambda}(\Omega)=1$, i.e. $c_{p}=1$. An explicit expression of $c_{\lambda}$ can be found in [17].

We write $\mathcal{A}_{\lambda}^{2}(\Omega)$ for the weighted Bergman space of holomorphic functions in $L^{2}\left(\Omega, d v_{\lambda}\right)$. The norm and inner product on these spaces are denoted by $\|\cdot\|_{\lambda}$ and $\langle\cdot, \cdot\rangle_{\lambda}$, respectively. The following result is well-known [16, 17]:

Lemma 2.1. The Bergman space $\mathcal{A}_{\lambda}^{2}(\Omega)$ is a reproducing kernel Hilbert space and the kernel can be expressed in terms of the Jordan triple determinant:

$$
K_{\lambda}(z, w)=h(z, w)^{-\lambda}, \quad \text { where } \quad(z, w) \in \Omega \times \Omega .
$$

We denote by $\beta_{\lambda}(\cdot, \cdot)$ the Bergman metric on $\Omega$ with respect to the weighted Bergman space $\mathcal{A}_{\lambda}^{2}(\Omega)$. More precisely, $\beta_{\lambda}$ is the metric distance function induced by the infinitesimal Bergman metric on $\Omega$ with metric tensor:

$$
\left(g_{i j}^{\lambda}(z)\right)_{i, j}=\left(\frac{\partial^{2}}{\partial z_{i} \partial \bar{z}_{j}} \log K_{\lambda}(z, z)\right)_{i, j} \in \mathbb{C}^{n \times n},
$$

where $K_{\lambda}$ denotes the reproducing kernel function as defined above. Then we have

$$
\beta_{\lambda}(z, w)=\sqrt{\frac{\lambda}{p}} \beta(z, w), \quad \text { with the definition } \quad \beta(z, w)=\beta_{p}(z, w) .
$$

2.1. Functions of bounded and vanishing oscillation. With fixed $w \in \Omega$ consider the normalized reproducing kernel $k_{w}^{\lambda} \in \mathcal{A}_{\lambda}^{2}(\Omega)$

$$
k_{w}^{\lambda}(z):=K_{\lambda}(z, w)\left\|K_{\lambda}(\cdot, w)\right\|_{\lambda}^{-1}=h(z, w)^{-\lambda} h(w, w)^{\frac{\lambda}{2}}, \quad z \in \Omega .
$$

The Berezin transform of $f \in L^{1}(\Omega, d v)$ (see [3, Lemma 4.1]) is the real analytic function on $\Omega$ defined by the integral transform

$$
\mathcal{B}_{\lambda}(f)(z):=\int_{\Omega} f(w)\left|k_{z}^{\lambda}(w)\right|^{2} d v_{\lambda}(w)
$$

(see (3.7) for yet another representation of the Berezin transform). Recall that the mean oscillation of $f \in L^{2}(\Omega, d v)$ at $z \in \Omega$ is given by:

$$
\mathrm{MO}^{\lambda}(f)(z):=\mathcal{B}_{\lambda}\left(|f|^{2}\right)(z)-\left|\mathcal{B}_{\lambda}(f)\right|^{2}(z)=\mathcal{B}_{\lambda}\left(\left|f-\mathcal{B}_{\lambda}(f)(z)\right|^{2}\right)(z) \geq 0 .
$$


We consider the family of semi-norms

$$
\|f\|_{\mathrm{BMO}^{\lambda}}:=\sup \left\{\sqrt{\mathrm{MO}^{\lambda}(f)(z)}: z \in \Omega\right\} .
$$

The space of functions having bounded $\lambda$-mean oscillation is given by:

$$
\operatorname{BMO}^{\lambda}(\Omega):=\left\{f: \Omega \rightarrow \mathbb{C}:\|f\|_{\mathrm{BMO}^{\lambda}}<\infty\right\} .
$$

In what follows we shortly write $\operatorname{BMO}(\Omega):=\mathrm{BMO}^{p}(\Omega)$ and $\mathrm{MO}(f):=\mathrm{MO}^{p}(f)$. Note that for all $\lambda>p-1$ :

$$
L^{\infty}(\Omega) \subsetneq \mathrm{BMO}^{\lambda}(\Omega) .
$$

Let $C_{0}(\Omega)=\left\{f \in C(\Omega): \lim _{z \rightarrow \partial \Omega} f(z)=0\right\}$ be the space of all continuous functions vanishing at the boundary $\partial \Omega$.

Definition 2.2. A complex valued function $g$ on $\Omega$ is said to have "vanishing mean oscillation" at $\partial \Omega$ if $\mathrm{MO}(g) \in C_{0}(\Omega)$. Put

$$
\mathrm{VMO}_{\partial}(\Omega):=\left\{g: \Omega \rightarrow \mathbb{C}: \operatorname{MO}(g) \in C_{0}(\Omega)\right\} .
$$

There is also the notion of bounded oscillation with respect to the weighted Bergman metric $\beta_{\lambda}$ :

Definition 2.3. Let $\lambda>p-1$. A continuous function $f$ is said to be of "bounded $\lambda$ oscillation" on $\Omega$ if

$$
\|f\|_{\mathrm{BO}^{\lambda}}:=\sup \left\{|f(z)-f(w)|: z, w \in \Omega, \beta_{\lambda}(z, w)<1\right\}<\infty .
$$

Clearly, one has for $\lambda \geq \mu$ :

$$
\|f\|_{\mathrm{BO}^{\lambda}} \leq\|f\|_{\mathrm{BO}^{\mu}} .
$$

We say that the function $f$ has "vanishing $\lambda$-oscillation at $\partial \Omega$ " if $\operatorname{Osc}_{z}^{\lambda}(f) \in C_{0}(\Omega)$, where

$$
\operatorname{Osc}_{z}^{\lambda}(f):=\sup \left\{|f(z)-f(w)|: w \in \Omega, \beta_{\lambda}(z, w)<1\right\}, \quad z \in \Omega,
$$

denotes the $\lambda$-oscillation of $f$ in $z$. We write $\mathrm{BO}^{\lambda}(\Omega)$ and $\mathrm{VO}_{\partial}^{\lambda}(\Omega)$ for the functions having bounded and vanishing $\lambda$-oscillation, respectively. For $\lambda=p$ we omit the superscript $p$ :

$$
\mathrm{BO}(\Omega):=\mathrm{BO}^{p}(\Omega) \quad \text { and } \quad \operatorname{VO}_{\partial}(\Omega):=\operatorname{VO}_{\partial}^{p}(\Omega) \text {. }
$$

By choosing a geodesic curve between two points $z, w \in \Omega$ and using (2.8) we obtain a global estimate:

Lemma 2.4. Let $f \in \mathrm{BO}^{\lambda}(\Omega)$. Then for all $w, z \in \Omega$ we have

$$
|f(z)-f(w)| \leq\|f\|_{\mathrm{BO}^{\lambda}}\left[1+\beta_{\lambda}(z, w)\right] .
$$

Proof. In case of $\beta_{\lambda}(z, w)<1$ we have (2.9) by definition. Otherwise we divide the geodesic curve in pieces and use the same calculation as in [26, Lemma 8.2, p. 209]. 
2.2. Toeplitz and Hankel operators. Our aim of this work is to analyze the asymptotic behavior of semi-commutators of Toeplitz operators when sending the weight parameter $\lambda>p-1$ to infinity. First we fix some basic notations. Consider the orthogonal projection

$$
P_{\lambda}: L^{2}\left(\Omega, d v_{\lambda}\right) \rightarrow \mathcal{A}_{\lambda}^{2}(\Omega) .
$$

Using Lemma 2.1, one can write $P_{\lambda}$ explicitly as

$$
\left(P_{\lambda} f\right)(z)=\int_{\Omega} f(w) h(z, w)^{-\lambda} d v_{\lambda}(w) .
$$

Given a symbol $f \in L^{\infty}(\Omega)$ we introduce the Toeplitz operator $T_{f}^{\lambda}$ and the Hankel operator $H_{f}^{\lambda}$ defined on $\mathcal{A}_{\lambda}^{2}(\Omega)$ by

$$
\begin{aligned}
T_{f}^{\lambda} & :=P_{\lambda} M_{f}, \\
H_{f}^{\lambda} & :=\left(I-P_{\lambda}\right) M_{f} .
\end{aligned}
$$

Here $M_{f}$ denotes the pointwise multiplication by $f$. A straightforward calculation shows the standard relation

$$
T_{f}^{\lambda} T_{g}^{\lambda}-T_{f g}^{\lambda}=-\left(H \frac{\lambda}{f}\right)^{*} H_{g}^{\lambda},
$$

which implies the norm estimate

$$
\left\|T_{f}^{\lambda} T_{g}^{\lambda}-T_{f g}^{\lambda}\right\|_{\lambda} \leq\left\|H \frac{\lambda}{f}\right\|_{\lambda}\left\|H_{g}^{\lambda}\right\|_{\lambda} .
$$

We are also concerned with Toeplitz operators having symbols in the space $\mathrm{UC}(\Omega)$ of complex valued functions on $\Omega$ that are uniformly continuous with respect to the Bergman metric distance $\beta$. Since $\mathrm{UC}(\Omega)$ contains unbounded functions (e.g. $f(z):=\beta(0, z)$ ) Toeplitz operators with uniformly continuous symbols are unbounded in general (cf. Remark 3.9] and [2]). Hence we need to define finite products of such operators in a careful way by specifying a common invariant dense domain.

We recall the Forelli-Rudin estimates [16, Proposition 8]: let the BSD $\Omega \subset \mathbb{C}^{n}$ be of type $(r, a, b)$ with characteristic multiplicities $a, b \in \mathbb{Z}_{+}$. Then we have:

Lemma 2.5. Let $\alpha>p-1$ and $t>\frac{r-1}{2} a$, then there is a constant $C>0$ (independent of $z \in \Omega$ ) such that for all $z \in \Omega$

$$
\int_{\Omega} h(w, w)^{\alpha-p}|h(z, w)|^{-(\alpha+t)} d v(w) \leq C h(z, z)^{-t} .
$$

Let $\rho>0$ and consider the following function spaces:

$$
S_{\rho}(\Omega):=\left\{f \in C(\Omega): \exists C>0 \text { s.t. }|f(z)| \leq C h(z, z)^{-\rho} \text { for all } z \in \Omega\right\} .
$$

Moreover, define $\mathcal{H}_{\rho}(\Omega):=S_{\rho}(\Omega) \cap \mathcal{A}_{\lambda}^{2}(\Omega)$ and consider the intersections

$$
\operatorname{Sym}(\Omega):=\bigcap_{\rho>0} S_{\rho}(\Omega) \quad \text { and } \quad \mathcal{D}:=\bigcap_{\rho>\rho^{*}} \mathcal{H}_{\rho}(\Omega),
$$


where $\rho^{*}:=\frac{r-1}{2} a$. Note that $\operatorname{Sym}(\Omega)$ is actually an algebra. Since $\mathcal{D}$ contains the restrictions of all holomorphic polynomials to $\Omega$ it is a dense subspace in all Bergman spaces $\mathcal{A}_{\lambda}^{2}(\Omega)$.

Lemma 2.6. Let $\lambda>\rho^{*}+p-1$ and assume that $f \in \operatorname{Sym}(\Omega)$. Then the (possibly unbounded) Toeplitz operator $T_{f}^{\lambda}$ leaves the space $\mathcal{D} \subset \mathcal{A}_{\lambda}^{2}(\Omega)$ invariant. In particular, all finite products

$$
T_{f_{1}}^{\lambda} T_{f_{2}}^{\lambda} \cdots T_{f_{m}}^{\lambda}: \mathcal{D} \longrightarrow \mathcal{D}
$$

with symbols $f_{j} \in \operatorname{Sym}(\Omega)$ are defined and induce densely defined operators on $\mathcal{A}_{\lambda}^{2}(\Omega)$.

Proof. Let $\rho \in\left(\rho^{*}, \lambda+1-p\right)$ and let $\varepsilon>0$ be sufficiently small such that $\alpha:=-\rho-\varepsilon+\lambda>$ $p-1$. Given $g \in \mathcal{D}$ and $f \in \operatorname{Sym}(\Omega)$ we can choose $C_{\rho}, c_{\varepsilon}>0$ such that

$$
|f(z)| \leq c_{\varepsilon} h(z, z)^{-\varepsilon} \quad \text { and } \quad|g(z)| \leq C_{\rho} h(z, z)^{-\rho} .
$$

The Forelli-Rudin estimates in Lemma 2.5 imply then:

$$
\begin{aligned}
\left|\left[T_{f}^{\lambda} g\right](z)\right| & =\left|\int_{\Omega} f(w) g(w) h(z, w)^{-\lambda} d v_{\lambda}(w)\right| \\
& \leq C_{\rho} c_{\varepsilon} \int_{\Omega} h(w, w)^{-\rho-\varepsilon+\lambda-p}|h(z, w)|^{-\lambda} d v(w) \\
& \leq C_{\rho} c_{\varepsilon} \int_{\Omega} h(w, w)^{\alpha-p}|h(z, w)|^{-(\alpha+\rho+\varepsilon)} d v(w) \\
& \leq \widetilde{C} C_{\rho} c_{\varepsilon} h(z, z)^{-(\rho+\varepsilon)} .
\end{aligned}
$$

Since $\rho>\rho^{*}$ and $\varepsilon>0$ can be chosen arbitrarily small, we conclude that $T_{f} g \in \mathcal{D}$.

Let $f \in \mathrm{BO}^{\lambda}(\Omega)$, then it follows from (2.9) with $z=0$ that

$$
|f(w)| \leq|f(0)|+|f(0)-f(w)| \leq|f(0)|+\|f\|_{\mathrm{BO}^{\lambda}}\left(1+\beta_{\lambda}(0, w)\right) .
$$

Corollary 3.3 below implies that for any $\rho>0$ there is $C(\rho, f)>0$ such that

$$
|f(w)| \leq C(\rho, f) h(w, w)^{-\rho}, \quad w \in \Omega,
$$

and therefore one obtains the inclusions

$$
\mathrm{UC}(\Omega) \subset \mathrm{BO}^{\lambda}(\Omega) \subset \operatorname{Sym}(\Omega) .
$$

Let $\mathcal{A}_{\text {uc }}(\Omega)$ denote the (non-closed) subalgebra in $\operatorname{Sym}(\Omega)$ which is generated by functions in $\mathrm{UC}(\Omega)$, i.e. $\mathcal{A}_{\mathrm{uc}}(\Omega)$ consists of finite sums of finite products of functions in $\mathrm{UC}(\Omega)$. Then we have:

Lemma 2.7. Toeplitz operators with symbols $f \in \mathcal{A}_{\mathrm{uc}}(\Omega)$ leave $\mathcal{D}$ invariant. In particular, finite products of such operators with dense domain $\mathcal{D}$ are well defined. 


\section{UNIFORMLY CONTINUOUS FUNCTIONS AND QUANTIZATION}

In the present section we study the asymptotic behavior of semi-commutators of Toeplitz operators with symbols in $f \in \mathrm{UC}(\Omega)$ (cf. Theorem 3.8). Although each single Toeplitz operator $T_{f}^{\lambda}$ may be unbounded it follows from the inclusions (2.11) together with the results in [5] that the semi-commutators $T_{g}^{\lambda} T_{f}^{\lambda}-T_{f g}^{\lambda}, f, g \in \mathrm{UC}(\Omega)$ are bounded operators. We start with some preparations (Lemma 3.1, 3.2 and Corollary 3.3), which give auxiliary inequalities that are essential in the proof of Proposition 3.4 devoted to the norm estimate of Hankel operators.

Lemma 3.1. Let $s, C_{1}, C_{2}>0, U \subset \mathbb{C}^{n}$ and let $f, g: U \rightarrow \mathbb{R}_{+}$satisfy $g(z) \geq 1$ and $f(z) \leq C_{1} g(z)$ for all $z \in U$. Further assume that there exists a set $V \subset U$ such that

$$
\begin{cases}f(z) \leq C_{2} \sqrt{\log g(z)} & \text { for all } z \in V \\ g(z) \geq 1+s & \text { for all } z \in U \backslash V .\end{cases}
$$

Then there exists a constant $C^{\prime}>0$ such that $\sqrt{\lambda} f(z) \leq C^{\prime} g(z)^{\lambda}$ for all $z \in U$ and $\lambda \geq 1$.

Proof. Fix $z \in V$ and $\lambda \geq 1$. If $g(z)=1$, then $f(z)=0$ and thus obviously $\sqrt{\lambda} f(z) \leq C^{\prime} g(z)^{\lambda}$ for all $\lambda \geq 1$ and any $C^{\prime}>0$. So assume that $g(z)>1$ and set $C_{V}^{\prime}:=\sqrt{\log 2} C_{2}$. Then

$$
C_{V}^{\prime} g(z)^{\lambda}=C_{V}^{\prime} 2^{\frac{\lambda \log g(z)}{\log 2}} \geq \sqrt{\frac{\log 2}{\log g(z)}} f(z) \sqrt{\frac{\lambda \log g(z)}{\log 2}}=\sqrt{\lambda} f(z)
$$

since $2^{y} \geq \sqrt{y}$ for all $y \geq 0$.

For $z \in U \backslash V$ we choose $C_{V^{c}}^{\prime}:=C_{1} \frac{1+s}{\sqrt{2 \log (1+s)}}$ so that

$$
C_{V^{c}}^{\prime} g(z)^{\lambda} \geq C_{1} \frac{(1+s)^{\lambda}}{\sqrt{2 \log (1+s)}} g(z) \geq C_{1} \sqrt{\lambda} g(z) \geq \sqrt{\lambda} f(z),
$$

where we used $\frac{(1+s)^{y}}{\sqrt{2 \log (1+s)}} \geq \sqrt{y}$ for all $y \geq 0$ and $s>0$. Choosing $C^{\prime}:=\max \left\{C_{V}^{\prime}, C_{V^{c}}^{\prime}\right\}$ finishes the proof.

Lemma 3.2. Let $\Omega \subset \mathbb{C}^{n}$ be a BSD and $\rho>0$. Then there is a neighborhood $V$ of 0 and a constant $C(\rho)>0$ such that

$$
\beta(0, z) \leq C(\rho) \sqrt{\log h(z, z)^{-\rho}}
$$

for all $z \in V$.

Proof. Since $\sqrt{\log h(z, z)^{-\rho}}=\sqrt{\rho} \sqrt{-\log h(z, z)}$, it clearly suffices to check the assertion for one particular $\rho$. We may thus assume that $\rho=\lambda>p-1$. 
With $s>0$ and each fixed $z \in \Omega$ consider the polynomial

$$
P_{z}(s):=h(s z, s z)=\prod_{j=1}^{r}\left(1-s^{2} t_{j}^{2}\right)=1-s^{2} \sum_{j=1}^{r} t_{j}^{2}+O\left(s^{4}\right) \quad(\text { as } s \downarrow 0) .
$$

Therefore the Taylor expansion in $z=0$ of $z \mapsto h(z, z)$ cannot have a linear term. Write

$$
h(z, z)=1+\sum_{|\alpha+\beta|>1} a_{\alpha \beta} z^{\alpha} \bar{z}^{\beta}
$$

and insert this expansion into (2.4):

$$
\log K_{\lambda}(z, z)=\log h(z, z)^{-\lambda}=-\lambda \log h(z, z)=-\lambda \log \left(1+\sum_{|\alpha+\beta|>1} a_{\alpha \beta} z^{\alpha} \bar{z}^{\beta}\right) .
$$

One obtains the Bergman metric tensor

$$
\begin{aligned}
g_{i j}(z):= & \frac{\partial^{2}}{\partial z_{i} \partial \bar{z}_{j}} \log K_{\lambda}(z, z) \\
=\frac{\lambda}{h(z, z)^{2}}\left(\sum_{|\alpha+\beta|>1} a_{\alpha \beta} \alpha_{i} z^{\alpha-e_{i}} \bar{z}^{\beta}\right) & \left(\sum_{|\alpha+\beta|>1} a_{\alpha \beta} \beta_{j} z^{\alpha} \bar{z}^{\beta-e_{j}}\right) \\
& -\frac{\lambda}{h(z, z)} \sum_{|\alpha+\beta|>1} a_{\alpha \beta} \alpha_{i} \beta_{j} z^{\alpha-e_{i}} \bar{z}^{\beta-e_{j}},
\end{aligned}
$$

where $e_{i}=(0, \ldots, 1, \ldots, 0)=\left(\delta_{i \ell}\right)_{\ell=1, \ldots, n} \in \mathbb{Z}_{+}^{n}$. For $z=0$ we have $\left(g_{i j}(0)\right)_{i j}=-\lambda\left(a_{e_{i} e_{j}}\right)_{i j}$. Since the metric tensor is positive definite, it follows that

$$
-A:=\left(a_{e_{i} e_{j}}\right)_{i j}<0 .
$$

Hence we can write the quadratic term in the expansion of (3.1) as follows:

$$
h(z, z)=1-\langle A z, z\rangle+\sum_{|\alpha+\beta|>2} a_{\alpha \beta} z^{\alpha} \bar{z}^{\beta} \quad \text { where } A=\frac{1}{\lambda}\left(g_{i j}(0)\right)_{i, j}>0 .
$$

Let $\mu>0$ denote the minimal eigenvalue of $\left(g_{i j}(0)\right)_{i j}$. Then we can choose a convex zero-neighborhood $V \subset \Omega$ such that

$$
\log h(z, z)^{-\lambda}=\log K_{\lambda}(z, z) \geq-\lambda \log \left(1-\frac{\mu}{2}|z|^{2}\right) \geq \frac{\lambda \mu}{2}|z|^{2}, \quad z \in V .
$$

Given $z \in V$ we can consider the straight path

$$
\gamma:[0,1] \rightarrow V: \gamma(t)=t z
$$


Then we can estimate:

$$
\beta(0, z) \leq \ell(\gamma)=\int_{0}^{1} \sqrt{\sum_{i, j=1}^{n} g_{i j}(t z) z_{i} \bar{z}_{j}} d t \leq \sup _{z \in V} \sqrt{\left\|\left(g_{i j}(z)\right)_{i j}\right\|} \cdot|z|=: C_{V}|z|,
$$

where $|z|^{2}:=\left|z_{1}\right|^{2}+\cdots+\left|z_{n}\right|^{2}$. A comparison of (3.3) and (3.4) gives for all $z \in V$ :

$$
\beta(0, z) \leq C_{V}|z| \leq \sqrt{\frac{2}{\lambda \mu}} C_{V} \sqrt{\log h(z, z)^{-\lambda}}=: C(\lambda) \sqrt{\log h(z, z)^{-\lambda}} .
$$

This finishes the proof.

Corollary 3.3. Let $\Omega \subset \mathbb{C}^{n}$ be a $B S D$ and $\rho>0$. Then there is a constant $C>0$ (depending only on $\rho$ ) such that

$$
\sqrt{\lambda} \beta(0, z) \leq C h(z, z)^{-\rho \lambda}
$$

for all $z \in \Omega$ and $\lambda \geq 1$.

Proof. It holds $h(z, z)^{-\rho} \geq 1$ and from [5, Equation (**) on p. 317] one has for all $z \in \Omega$ :

$$
\beta(0, z) \leq C_{1} h(z, z)^{-\rho} .
$$

Furthermore, Lemma 3.2 implies $\beta(0, z) \leq C_{2} \sqrt{\log h(z, z)^{-\rho}}$ for all $z$ in a suitable zeroneighborhood $V \subset \Omega$. The product form (2.2) shows that $h(z, z)^{-\rho}>1$ for $z \neq 0$ and $h(z, z)^{-\rho} \rightarrow \infty$ as $z \rightarrow \partial \Omega$. Therefore one also has

$$
h(z, z)^{-\rho} \geq 1+s
$$

for some $s>0$ and all $z \in \Omega \backslash V$. Setting $f(z):=\beta(0, z)$ and $g(z):=h(z, z)^{-\rho}$, the result follows from Lemma 3.1 .

Proposition 3.4. Let $f \in \mathrm{BO}^{\lambda}(\Omega)$. Then there is a constant $C>0$, independent of $f$ and of $\lambda>4 p$, such that

$$
\left\|H_{f}^{\lambda}\right\|_{\lambda} \leq C\|f\|_{\mathrm{BO}^{\lambda}} .
$$

Proof. Let $g \in \mathcal{A}_{\lambda}^{2}(\Omega)$. From the integral expression of the Hankel operator

$$
\left[H_{f}^{\lambda} g\right](z)=\int_{\Omega}[f(z)-f(w)] g(w) K_{\lambda}(z, w) d v_{\lambda}(w), \quad z \in \Omega,
$$

and the estimate (2.9) in Lemma 2.4 it follows that

$$
\left|H_{f}^{\lambda} g(z)\right| \leq\|f\|_{\mathrm{BO}^{\lambda}} \int_{\Omega}\left(\beta_{\lambda}(z, w)+1\right)|h(z, w)|^{-\lambda}|g(w)| d v_{\lambda}(w) .
$$

The constant $C>0$ can be chosen as the norm of the integral operator

$$
L^{2}\left(\Omega, d v_{\lambda}\right) \ni u \mapsto \mathcal{L}_{\lambda}(u)(z):=\int_{\Omega} \underbrace{\left(\beta_{\lambda}(z, w)+1\right)|h(z, w)|^{-\lambda}}_{=: L_{\lambda}(z, w)} u(w) d v_{\lambda}(w) \in L^{2}\left(\Omega, d v_{\lambda}\right) .
$$


In order to estimate the norm (independently of $\lambda$ ) we apply the Schur test. Since the integral kernel $L_{\lambda}(z, w)$ of $\mathcal{L}_{\lambda}$ is symmetric in $z$ and $w$ it is sufficient to construct a positive function $h$ on $\Omega$ and a constant $C>0$ independent of $\lambda$ such that for all $z \in \Omega$ :

$$
I_{\lambda}(z):=\int_{\Omega}\left(\beta_{\lambda}(z, w)+1\right)|h(z, w)|^{-\lambda} h(w) d v_{\lambda}(w) \leq C h(z) .
$$

With $t$ to be determined later put

$$
h(z):=h(z, z)^{t},
$$

and let $\varphi_{z}$ be an involutive automorphism of $\Omega$ interchanging 0 and $z$. A change of variables and the identity $\beta_{\lambda}(z, w)=\beta_{\lambda}\left(0, \varphi_{z}(w)\right)$ gives:

$$
\begin{aligned}
I_{\lambda}(z) & =c_{\lambda} \int_{\Omega}\left[\beta_{\lambda}\left(0, \varphi_{z}(w)\right)+1\right]|h(z, w)|^{-\lambda} h(w, w)^{t+\lambda-p} d v(w) \\
& =c_{\lambda} \int_{\Omega}\left[\beta_{\lambda}(0, w)+1\right]\left|h\left(z, \varphi_{z}(w)\right)\right|^{-\lambda} h\left(\varphi_{z}(w), \varphi_{z}(w)\right)^{t+\lambda-p} d v\left(\varphi_{z}(w)\right)=(+) .
\end{aligned}
$$

We can use the following standard relations (see e.g. [16])

$$
h\left(z, \varphi_{z}(w)\right)=\frac{h(z, z)}{h(z, w)} \quad \text { and } \quad h\left(\varphi_{z}(w), \varphi_{z}(w)\right)=\frac{h(z, z) h(w, w)}{|h(z, w)|^{2}},
$$

as well as

$$
d v\left(\varphi_{z}(w)\right)=\left(\frac{h(z, z)}{|h(z, w)|^{2}}\right)^{p} d v(w)
$$

to obtain

$$
(+)=c_{\lambda} \underbrace{h(z, z)^{t}}_{=h(z)} \int_{\Omega}\left[\beta_{\lambda}(0, w)+1\right] h(w, w)^{t+\lambda-p}|h(z, w)|^{-2 t-\lambda} d v(w) .
$$

Now we apply Corollary 3.3. For any $\rho>0$ there is a constant $C=C(\rho)>0$ (independent of $\lambda$ and $w \in \Omega$ ) such that

$$
1+\beta_{\lambda}(0, w)=1+\sqrt{\frac{\lambda}{p}} \beta(0, w) \leq C(\rho) h(w, w)^{-\rho \lambda} .
$$

Hence we can further estimate $(+)$ by

$$
(+) \leq c_{\lambda} C(\rho) h(z) \int_{\Omega} h(w, w)^{t+(1-\rho) \lambda-p}|h(z, w)|^{-2 t-\lambda} d v(w)=(++) .
$$

Choosing $t=-\frac{\lambda}{2}$ and $\rho=\frac{1}{4}$, we obtain

$$
(++)=c_{\lambda} C\left(\frac{1}{4}\right) h(z) \int_{\Omega} h(w, w)^{\frac{\lambda}{4}-p} d v(w)=\frac{c_{\lambda}}{c_{\frac{\lambda}{4}}} C\left(\frac{1}{4}\right) h(z) .
$$


Since $\frac{c_{\lambda}}{c_{\frac{\lambda}{4}}}$ is bounded (as a function of $\lambda$, cf. [17]), the result follows from the Schur test.

A relation between $\operatorname{BMO}^{\lambda}(\Omega)$ and $\mathrm{BO}^{\lambda}(\Omega)$ is given by the following result:

Theorem 3.5. Let $g \in \mathrm{BMO}^{\lambda}(\Omega)$ and $\lambda \geq p$. Then we have for all $z, w \in \Omega$

$$
\left|\mathcal{B}_{\lambda}(g)(w)-\mathcal{B}_{\lambda}(g)(z)\right| \leq 2\|g\|_{\mathrm{BMO}^{\lambda}} \beta_{\lambda}(z, w) .
$$

In particular, $\mathcal{B}_{\lambda}(g) \in \mathrm{BO}^{\lambda}(\Omega)$ and

$$
\left\|\mathcal{B}_{\lambda}(g)\right\|_{\mathrm{BO}^{\lambda}} \leq 2\|g\|_{\mathrm{BMO}^{\lambda}}
$$

Proof. See [3, Theorem 4.9].

Let $\lambda>4 p$, replace $f \in \mathrm{BO}^{\lambda}(\Omega)$ in Proposition 3.4 by $\mathcal{B}_{\lambda}(f)$, where $f \in \mathrm{BMO}^{\lambda}(\Omega)$, and use Theorem 3.5. We obtain a constant $C>0$ independent of $f$ and $\lambda$ such that

$$
\left\|H_{\mathcal{B}_{\lambda}(f)}^{\lambda}\right\|_{\lambda} \leq C\left\|\mathcal{B}_{\lambda}(f)\right\|_{\mathrm{BO}^{\lambda}} \leq 2 C\|f\|_{\mathrm{BMO}^{\lambda}} .
$$

In particular, let $f \in \mathrm{UC}(\Omega)$ be uniformly continuous w.r.t. the Bergman metric $\beta(z, w)$. We analyze the asymptotic behavior of $\|f\|_{\mathrm{BMO}^{\lambda}}$ as $\lambda \rightarrow \infty$. By applying a change of variables in the integral, the Berezin transform (2.6) of a function $f$ can be represented as a convolution type integral (cf. [3]):

$$
\mathcal{B}_{\lambda}(f)(x)=c_{\lambda} \int_{\Omega}\left(f \circ \varphi_{x}\right)(y) h(y, y)^{\lambda-p} d v(y) .
$$

We will use the following asymptotic behavior of the Berezin transform for uniformly continuous symbols:

Proposition 3.6. Let $\Omega \subset \mathbb{C}^{n}$ be a $B S D$ and $f \in \mathrm{UC}(\Omega)$. Then

$$
\lim _{\lambda \rightarrow \infty} \mathcal{B}_{\lambda}(f)=f
$$

where the convergence is uniformly on $\Omega$.

Proof. See [3, Proposition 4.4].

According to (2.7) and (3.7), we can write

$$
\begin{aligned}
\mathrm{MO}^{\lambda}(f)(x) & =c_{\lambda} \int_{\Omega}\left|f \circ \varphi_{x}(y)-\mathcal{B}_{\lambda}(f)(x)\right|^{2} h(y, y)^{\lambda-p} d v(y) \\
& =c_{\lambda} \int_{\Omega}\left|f \circ \varphi_{x}(y)-\mathcal{B}_{\lambda}(f) \circ \varphi_{x}(0)\right|^{2} h(y, y)^{\lambda-p} d v(y) .
\end{aligned}
$$

The next observation is crucial in the proof of our main theorem:

Proposition 3.7. Let $f \in \mathrm{UC}(\Omega)$. Then $\lim _{\lambda \rightarrow \infty}\|f\|_{\mathrm{BMO}^{\lambda}}=0$. 
Proof. Let $\frac{1}{4}>\varepsilon>0$ be fixed and choose $\delta>0$ such that $|f(z)-f(w)|<\varepsilon$ for all $z, w \in \Omega$ with $\beta(z, w)<\delta$. We divide the domain of integration into two parts:

$$
\mathrm{MO}^{\lambda}(f)(x)=c_{\lambda}\left\{\int_{\beta(y, 0)<\delta}+\int_{\beta(y, 0) \geq \delta}\right\}\left|f \circ \varphi_{x}(y)-\mathcal{B}_{\lambda}(f) \circ \varphi_{x}(0)\right|^{2} h(y, y)^{\lambda-p} d v(y) .
$$

In the case $\beta(y, 0)<\delta$ we have

$$
\beta\left(\varphi_{x}(y), \varphi_{x}(0)\right)=\beta(y, 0)<\delta
$$

uniformly for all $x \in \Omega$. The uniform continuity of $f$ and Proposition 3.6 for sufficiently large weight parameter $\lambda$ imply then that

$$
\begin{aligned}
\left|f \circ \varphi_{x}(y)-\mathcal{B}_{\lambda}(f) \circ \varphi_{x}(0)\right|^{2} & \leq \\
& \leq\left(\left|f \circ \varphi_{x}(y)-f \circ \varphi_{x}(0)\right|+\left|\left(f-\mathcal{B}_{\lambda}(f)\right) \circ \varphi_{x}(0)\right|\right)^{2}<4 \varepsilon^{2}<\varepsilon .
\end{aligned}
$$

Hence we obtain

$$
0 \leq \mathrm{MO}^{\lambda}(f)(x) \leq \varepsilon+c_{\lambda} \int_{\beta(y, 0) \geq \delta}\left|f \circ \varphi_{x}(y)-\mathcal{B}_{\lambda}(f) \circ \varphi_{x}(0)\right|^{2} h(y, y)^{\lambda-p} d v(y)=(+) .
$$

It is known (see [3, Lemma 2.1] or (2.11)) that $\mathrm{UC}(\Omega) \subset \mathrm{BO}(\Omega)$ and therefore

$$
\begin{aligned}
\left|f \circ \varphi_{x}(y)-f \circ \varphi_{x}(0)\right| & \leq\|f\|_{\mathrm{BO}^{p}}\left[1+\beta_{p}\left(\varphi_{x}(y), \varphi_{x}(0)\right)\right] \\
& =\|f\|_{\mathrm{BO}^{p}}[1+\beta(y, 0)] .
\end{aligned}
$$

The difference $f-\mathcal{B}_{\lambda}(f)$ is uniformly bounded on $\Omega$. According to Proposition [3.6, we have

$$
\lim _{\lambda \rightarrow \infty}\left\|f-\mathcal{B}_{\lambda}(f)\right\|_{\infty}=0
$$

and therefore it follows for all $x \in \Omega$ and sufficiently large parameter $\lambda$ that

$$
\left|f \circ \varphi_{x}(y)-\mathcal{B}_{\lambda}(f) \circ \varphi_{x}(0)\right| \leq 2\|f\|_{\mathrm{BO}^{p}}[1+\beta(y, 0)] .
$$

The last estimate implies that for sufficiently large $\lambda$ and all $x \in \Omega$ we have

$$
(+) \leq \varepsilon+4 c_{\lambda}\|f\|_{\mathrm{BO}^{p}}^{2} \int_{\beta(y, 0) \geq \delta}[1+\beta(y, 0)]^{2} h(y, y)^{\lambda-p} d v(y) .
$$

Since $g(y):=1+\beta(y, 0)$ defines an element in $L^{2}(\Omega, d v)$ (see [5, Theorem E]), $c_{\lambda} \sim \lambda^{n}$ as $\lambda \rightarrow \infty$ (see e.g. [3]) and there is a constant $s>0$ (only depending on $\delta$ ) such that $h(z, z) \leq 1-s$ for all $z \in \Omega$ with $\beta(z, 0) \geq \delta$, it follows that

$$
\lim _{\lambda \rightarrow \infty} 4 c_{\lambda}\|f\|_{\mathrm{BO}^{p}}^{2} \int_{\beta(y, 0) \geq \delta}[1+\beta(y, 0)]^{2} h(y, y)^{\lambda-p} d v(y)=0 .
$$

This implies that $\lim _{\lambda \rightarrow \infty}\|f\|_{\mathrm{BMO}^{\lambda}}=0$. 
By combining the previous estimates, we obtain the following quantization result on the semi-commutator of Toeplitz operators with symbols in $\mathrm{UC}(\Omega)$.

Theorem 3.8. Let $f \in \mathrm{UC}(\Omega)$. Then $\lim _{\lambda \rightarrow \infty}\left\|H_{f}^{\lambda}\right\|_{\lambda}=0$. In particular,

$$
\lim _{\lambda \rightarrow \infty}\left\|T_{f}^{\lambda} T_{g}^{\lambda}-T_{f g}^{\lambda}\right\|_{\lambda}=0
$$

for all $g \in L^{\infty}(\Omega)$ or all $g \in \mathrm{UC}(\Omega)$.

Proof. According to (2.10), it is sufficient to show that $\lim _{\lambda \rightarrow \infty}\left\|H_{f}^{\lambda}\right\|_{\lambda}=0$. We use the estimate:

$$
\left\|H_{f}^{\lambda}\right\|_{\lambda} \leq\left\|H_{f-\mathcal{B}_{\lambda}(f)}^{\lambda}\right\|_{\lambda}+\left\|H_{\mathcal{B}_{\lambda}(f)}^{\lambda}\right\|_{\lambda} \leq\left\|f-\mathcal{B}_{\lambda}(f)\right\|_{\infty}+\left\|H_{\mathcal{B}_{\lambda}(f)}^{\lambda}\right\|_{\lambda} .
$$

From Proposition 3.6 we conclude that the first summand on the right-hand side tends to zero as $\lambda \rightarrow \infty$. Proposition 3.7 together with estimate (3.6) implies that $\lim _{\lambda \rightarrow \infty}\left\|H_{\mathcal{B}_{\lambda}(f)}^{\lambda}\right\|_{\lambda}=0$, and the assertion follows.

Remark 3.9. As was previously mentioned, the space $\mathrm{UC}(\Omega)$ contains unbounded functions. In [2, Theorem 3.8] the following equivalence is shown for Toeplitz operators with symbols in $\mathrm{UC}(\Omega)$ :

$$
T_{f} \text { is bounded, } f \in \mathrm{UC}(\Omega) \quad \Longleftrightarrow \quad f \in \mathrm{UC}(\Omega) \cap L^{\infty}(\Omega) .
$$

According to (2.11), we have the inclusions

$$
\mathrm{UC}(\Omega) \subset \mathrm{BO}^{\lambda}(\Omega) \subset \mathrm{BMO}^{\lambda}(\Omega) .
$$

Moreover, $H_{f}^{\lambda}$ is bounded in case of $f \in \mathrm{BMO}^{\lambda}(\Omega)$. In particular, the Hankel operator $H_{f}^{\lambda}$ with uniformly continuous symbol $f$ is bounded. Therefore the semi-commutators in (3.10) are bounded although each single Toeplitz operator may be unbounded (cf. Section 2.2).

If one prefers to deal with bounded Toeplitz operators, one may choose the symbols from the space $\left(C^{*}\right.$-algebra) $\mathrm{BUC}(\Omega)$ of bounded $\beta$-uniformly continuous functions on $\Omega$. Note that

$$
C(\bar{\Omega}) \subsetneq \mathrm{BUC}(\Omega) \text {. }
$$

In this case a stronger version of Theorem 3.8 holds true:

Corollary 3.10. Let $f_{1}, \cdots f_{m} \in \operatorname{BUC}(\Omega)$. Then we have

$$
\lim _{\lambda \rightarrow \infty}\left\|T_{f_{1}}^{\lambda} T_{f_{2}}^{\lambda} \cdots T_{f_{m}}^{\lambda}-T_{f_{1} f_{2} \cdots f_{m}}^{\lambda}\right\|_{\lambda}=0 .
$$

Proof. Use Theorem 3.8 and standard estimates.

We draw some further conclusions and comment on the compactness of semi-commutators.

Lemma 3.11. Let $\Omega$ be a $B S D$ in $\mathbb{C}^{n}$, then $\operatorname{VMO}_{\partial}(\Omega) \cap \mathrm{UC}(\Omega)=\operatorname{VO}_{\partial}(\Omega)$. 
Proof. Let $f \in \mathrm{VO}_{\partial}(\Omega)$. Given $\varepsilon>0$, select a compact subset $K$ of $\Omega$ such that for each $z \in \Omega \backslash K$ we have that

$$
\operatorname{Osc}_{z}^{p}(f)=\sup \{|f(z)-f(w)|: w \in \Omega, \beta(z, w)<1\}<\varepsilon .
$$

The restriction $\left.f\right|_{K}$ is obviously uniformly continuous. Thus we can find $\delta$, which depends on the given $\varepsilon$ so that $|f(z)-f(w)|<\varepsilon$ whenever $\beta(z, w)<\delta$. That is $\operatorname{VO}_{\partial}(\Omega) \subset \mathrm{UC}(\Omega)$.

Furthermore, by [5, Theorem B],

$$
\operatorname{VMO}_{\partial}(\Omega)=\operatorname{VO}_{\partial}(\Omega)+\mathcal{J},
$$

where, by [9, pages 940 and 944], $\mathcal{J}$ consists of all functions $g \in \mathrm{VMO}_{\partial}(\Omega)$ such that the Toeplitz operator $T_{g}$ is compact. Now, $\mathrm{VO}_{\partial}(\Omega) \subset \mathrm{UC}(\Omega)$ implies that

$$
\operatorname{VMO}_{\partial}(\Omega) \cap \mathrm{UC}(\Omega)=\operatorname{VO}_{\partial}(\Omega)+\mathcal{J} \cap \mathrm{UC}(\Omega) .
$$

By [2, Theorem 3.8], $\mathcal{J} \cap \mathrm{UC}(\Omega)=C_{0}(\Omega) \subset \operatorname{VO}_{\partial}(\Omega)$, which finishes the proof.

Consider the function space

$$
\Gamma:=\left\{f \in L^{\infty}(\Omega): T_{g}^{\lambda} T_{f}^{\lambda}-T_{g f}^{\lambda} \text { is compact for all } g \in L^{\infty}(\Omega)\right\} .
$$

We summarize the results of [5, Theorem B, Section 9], [9, Theorem A, Proposition 1], and [28, Proposition 6] in the following statement. Note that, although the paper [28] is devoted to the case of the unit disk, the result of its Proposition 6 remains valid for the case of a general bounded symmetric domain $\Omega$.

Theorem 3.12. Let $f \in L^{\infty}(\Omega)$. Then the following statements are equivalent:

(1) $f \in \Gamma \cap \bar{\Gamma}$,

(2) $f \in \mathrm{VO}_{\partial}(\Omega)+\mathcal{J}$,

(3) $H_{f}$ and $H_{\bar{f}}$ are compact,

(4) $\left[P_{\lambda}, M_{f}\right]$ is compact,

(5) $T_{f}^{\lambda} T_{\bar{f}}^{\lambda}-T_{|f|^{2}}^{\lambda}$ and $T_{\bar{f}}^{\lambda} T_{f}^{\lambda}-T_{|f|^{2}}^{\lambda}$ are compact.

Thus the operator-theoretic version of Lemma 3.11 reads as follows.

Corollary 3.13. Let $f \in \mathrm{BUC}(\Omega)$. Then (i) and (ii) are equivalent:

(i) Both semi-commutators $T_{f}^{\lambda} T_{g}^{\lambda}-T_{f g}^{\lambda}$ and $T_{g}^{\lambda} T_{f}^{\lambda}-T_{g f}^{\lambda}$ are compact for all $g \in L^{\infty}(\Omega)$,

(ii) $f \in \mathrm{VO}_{\partial}(\Omega)$.

We mention (cf. [9, page 924]) that, in the case of $\Omega=\mathbb{B}^{n}$, the algebra $C\left(\overline{\mathbb{B}^{n}}\right)$ is a subset of $\operatorname{VO}_{\partial}\left(\mathbb{B}^{n}\right)$, and that this inclusion fails for higher rank domains. That is, in the classical situation of $\Omega=\mathbb{B}^{n}$ and $f \in C\left(\overline{\mathbb{B}^{n}}\right)$, the semi-commutator

$$
T_{f}^{\lambda} T_{g}^{\lambda}-T_{f g}^{\lambda}
$$

of Theorem 3.8 is compact for each $g \in L^{\infty}\left(\mathbb{B}^{n}\right)$ and all $\lambda>p-1=n$. However, in case of operator symbols from $\mathrm{BUC}(\Omega)$ such compactness does not need to be true. 
EXAMPLE 3.14. Let $\Omega=\mathbb{D}$ be the unit disk. Given a point $t_{0}$ on the unit circle $S^{1}=\partial \mathbb{D}$, let $\ell_{t_{0}}$ be the arc on $S^{1}$ with endpoint $t_{0}$ and $-t_{0}$. Define then the function

$$
f_{t_{0}}(z)=2 \omega\left(z, \ell_{t_{0}}, \mathbb{D}\right)-1, \quad z \in \mathbb{D},
$$

where

$$
\omega\left(z, \ell_{t_{0}}, \mathbb{D}\right)=\int_{\ell_{t_{0}}} \frac{1-|z|^{2}}{\left|e^{i \theta}-z\right|^{2}} \frac{d \theta}{2 \pi}
$$

is the harmonic measure of $\ell_{t_{0}}$ at $z$ in $\mathbb{D}$, cf. [18, page 5]. The function $f_{\ell_{t_{0}}}$, being bounded and harmonic, belongs to $\mathrm{BUC}(\mathbb{D})$. At the same time $f_{\ell_{t_{0}}}$ is a boundary piecewise continuous function in the sense of [25, Section 5].

Theorem 5.1 of [25] describes the quotient algebra of the algebra generated by Toeplitz operators with boundary piecewise continuous symbols modulo compact operators. This description implies that the self semi-commutator $T_{f_{t_{0}}}^{\lambda} T_{f_{t_{0}}}^{\lambda}-T_{f_{t_{0}}^{2}}^{\lambda}$ is not compact, while for any point $t_{1} \in S^{1}$ different from $\pm t_{0}$, the semi-commutator $T_{f_{t_{0}}}^{\lambda} T_{f_{t_{1}}}^{\lambda}-T_{f_{t_{0}} f_{t_{1}}}^{\lambda}$ is compact.

Note that the paper [25] deals with the classical Bergman space $(\lambda=p)$, but the results therein remain valid (with correspondent adjustments in formulas) for each weighted Bergman space $\mathcal{A}_{\lambda}^{2}(\mathbb{D})$ with $\lambda>p-1$.

The following example shows that Theorem 3.8 may fail for bounded symbols being not continuous in $\Omega$ (cf. [1, Example 5.1]).

ExAmple 3.15. We consider the unit disc $\Omega=\mathbb{D}$ in $\mathbb{C}$. Let $f \in L^{\infty}(\mathbb{D})$ be defined by

$$
f(z):= \begin{cases}1 & \text { if } z=0 \\ e^{i|z|^{-2}} & \text { if } z \neq 0 .\end{cases}
$$

Since $\bar{f} f=1$, we get $T_{\bar{f} f}^{\lambda}=$ Id for all $\lambda=2+\alpha$, where $\alpha>-1$ (see below). We will now show that $T_{f}^{\lambda} 1 \rightarrow 0$ as $\lambda \rightarrow \infty$, which contradicts the statement of Theorem 3.8. Since $f$ is radially symmetric, [19, Theorem 3.1] implies

$$
\left(T_{f}^{\lambda} 1\right)(z)=(\alpha+1) \int_{0}^{1} e^{i r^{-1}}(1-r)^{\alpha} d r=\frac{\alpha+1}{\alpha} \int_{\frac{1}{\alpha}}^{\infty} e^{i \alpha x}\left(1-\frac{1}{\alpha x}\right)^{\alpha} \frac{1}{x^{2}} d x=(+)
$$

where we have used the substitution $x:=\frac{1}{\alpha} r^{-1}$ (for $\alpha \geq 1$, say). We change variables again and put $y:=x+\frac{\pi}{\alpha}$. This yields

$$
(+)=-\frac{\alpha+1}{\alpha} \int_{\frac{1+\pi}{\alpha}}^{\infty} e^{i \alpha y}\left(1-\frac{1}{\alpha y-\pi}\right)^{\alpha} \frac{1}{\left(y-\frac{\pi}{\alpha}\right)^{2}} d y .
$$

By taking the average of (3.11) and (3.12), we get

$$
\left(T_{f}^{\lambda} 1\right)(z)=\frac{\alpha+1}{2 \alpha} \int_{0}^{\infty} e^{i \alpha x}\left[\left(1-\frac{1}{\alpha x}\right)^{\alpha} \frac{\chi_{\left[\frac{1}{\alpha}, \infty\right)}(x)}{x^{2}}-\left(1-\frac{1}{\alpha x-\pi}\right)^{\alpha} \frac{\chi_{\left[\frac{1+\pi}{\alpha}, \infty\right)}(x)}{\left(x-\frac{\pi}{\alpha}\right)^{2}}\right] d x .
$$


The integrand on the right-hand side is uniformly bounded by

$$
e^{-\frac{1}{x}} \frac{1}{x^{2}}+e^{-\frac{1}{x}} \min \left\{1, \frac{1}{(x-\pi)^{2}}\right\} \in L^{1}([0, \infty))
$$

for $\alpha=\lambda-2 \geq 1$ and converges pointwise to 0 . Thus $T_{f}^{\lambda} 1 \rightarrow 0$ as $\lambda \rightarrow \infty$ by the dominated convergence theorem.

\section{QuAntization AND "VMO INSIDE"}

Let $\Omega=\mathbb{B}^{n} \subset \mathbb{C}^{n}$ denote the open Euclidean unit ball. In this case the genus is $p=n+1$ and the rank $r=1$. As usual, we put $\lambda=n+1+\alpha$, where $\alpha>-1$. With our previous notation and $\lambda>n=p-1$ the weighted measure on $\mathbb{B}^{n}$ is given by

$$
d v_{\lambda}(y)=c_{\lambda} h(y, y)^{\lambda-p} d v(y)=\frac{\Gamma(n+1+\alpha)}{n ! \Gamma(\alpha+1)}\left(1-|y|^{2}\right)^{\alpha} d v(y)
$$

With $\rho>0$ and $x \in \mathbb{B}^{n}$ consider the Bergman balls

$$
E(x, \rho):=\left\{y \in \mathbb{B}^{n}: \beta(x, y)<\rho\right\} \quad \text { with volume }|E(x, \rho)|=\int_{E(x, \rho)} 1 d v(y) .
$$

For a locally integrable function $f$ on $\mathbb{B}^{n}$ and by using the notation in [9] we define the averaging function

$$
\hat{f}(x, \rho):=\frac{1}{|E(x, \rho)|} \int_{E(x, \rho)} f(y) d v(y) .
$$

With $q \geq 1$ and $f \in L^{q}\left(\mathbb{B}^{n}\right)$ put now

$$
A_{q}(f, \rho, x):=\frac{1}{|E(x, \rho)|} \int_{E(x, \rho)}|f(y)-\hat{f}(x, \rho)|^{q} d v(y) .
$$

Definition 4.1. With $q=2$ we define the space of bounded functions that have vanishing oscillation inside the unit ball (cf. [28])

$$
\operatorname{VMO}\left(\mathbb{B}^{n}\right):=\left\{f \in L^{\infty}\left(\mathbb{B}^{n}\right): \lim _{\rho \rightarrow 0} A_{2}(f, \rho, x)=0 \text { uniformly for } x \in \mathbb{B}^{n}\right\} .
$$

Note that different from standard notations we assume functions in $\operatorname{VMO}\left(\mathbb{B}^{n}\right)$ to be bounded.

Remark 4.2. A standard estimate shows that

$$
A_{2}(f, \rho, x) \leq \frac{1}{|E(x, \rho)|^{2}} \int_{E(x, \rho) \times E(x, \rho)}|f(y)-f(z)|^{2} d v(y, z) .
$$

Note that one has the proper inclusion $\operatorname{BUC}\left(\mathbb{B}^{n}\right) \subsetneq \operatorname{VMO}\left(\mathbb{B}^{n}\right)$. Here is an example of a function in $\operatorname{VMO}(\mathbb{D})$ that is not continuous: 
EXAMPLE 4.3. Let $\Omega=\mathbb{D}=\mathbb{B}^{1}$ and with $r \in(0,1)$ put

$$
f(r):=\log \left(\log \left(1+\frac{1}{r}\right)\right)
$$

Consider $g=f \circ|\cdot|: \mathbb{D} \backslash\{0\} \rightarrow \mathbb{C}$. This function is clearly not continuous at 0 . Moreover, $f$ is convex and

$$
\left|f^{\prime}(r)\right|=\left|\frac{1}{\log \left(1+\frac{1}{r}\right)} \frac{1}{1+\frac{1}{r}} \frac{1}{r^{2}}\right| \leq \frac{1}{r \log \left(1+\frac{1}{r}\right)} .
$$

Thus

$$
\begin{aligned}
A_{2}(g, \rho, x) & \leq \frac{1}{|E(x, \rho)|^{2}} \int_{E(x, \rho)^{2}}|f(|y|)-f(|z|)|^{2} d v(y, z) \\
& =\frac{2}{|E(x, \rho)|^{2}} \int_{\substack{E(x, \rho)^{2} \\
|y| \leq|z|}}|f(|y|)-f(|z|)|^{2} d v(y, z) \\
& \leq \frac{2}{|E(x, \rho)|^{2}} \int_{\substack{E(x, \rho)^{2} \\
|y| \leq|z|}}\left|f^{\prime}(|y|)\right|^{2} \underbrace{(|y|-|z|)^{2}}_{\leq \frac{4}{\pi}|E(x, \rho)|} d v(y, z) \\
& \leq \frac{8}{\pi} \int_{E(x, \rho)} \frac{1}{\left(|y| \log \left(1+\frac{1}{|y|}\right)\right)^{2}} d v(y) .
\end{aligned}
$$

Since $\frac{1}{\left(|y| \log \left(1+\frac{1}{|y|}\right)\right)^{2}}$ is integrable on $\mathbb{D}, A_{2}(g, \rho, x)$ tends uniformly to 0 as $\rho \rightarrow 0$. Of course, $g$ is unbounded. To get an example of a bounded function, just consider sin $\mathrm{g}$.

With $\lambda>p-1$ and the involution $\varphi_{x}$ consider the mean oscillation in (2.7) again:

$$
\mathrm{MO}^{\lambda}(f)(x)=\int_{\mathbb{B}^{n}}\left|f \circ \varphi_{x}(y)-\mathcal{B}_{\lambda}(f)(x)\right|^{2} d v_{\lambda}(y) .
$$

Lemma 4.4. For all $x \in \mathbb{B}^{n}$ and all parameters $\rho>0, \lambda>n$ we have

$$
\operatorname{MO}^{\lambda}(f)(x) \leq \int_{\mathbb{B}^{n}}\left|f \circ \varphi_{x}(y)-\hat{f}(x, \rho)\right|^{2} d v_{\lambda}(y) .
$$

Proof. Let $x \in \mathbb{B}^{n}$ and fix the parameters $\rho>0$ and $\lambda>p-1=n$. Consider the function $L: \mathbb{C} \rightarrow \mathbb{R}_{+}$defined by

$$
L(c):=\int_{\mathbb{B}^{n}}\left|f \circ \varphi_{x}(y)-c\right|^{2} d v_{\lambda}(y) .
$$

Then the gradient grad $L(c)$ vanishes precisely for

$$
c=\int_{\mathbb{B}^{n}} f \circ \varphi_{x}(y) d v_{\lambda}(y)=\mathcal{B}_{\lambda}(f)(x) .
$$

Since $L$ attains a minimum in the complex plane, the assertion follows. 
Theorem 4.5. Let $f \in \operatorname{VMO}\left(\mathbb{B}^{n}\right)$, then $\lim _{\lambda \rightarrow \infty}\|f\|_{\mathrm{BMO}^{\lambda}}=0$.

Proof. Let $t>0$ be a parameter which we will specify later on, $\lambda \geq p$ and put $\rho(\lambda):=c_{\lambda}^{-\frac{1}{2 n}}$. The estimate in Lemma 4.4 shows that:

$$
\begin{aligned}
\mathrm{MO}^{\lambda}(f)(x) & \leq\left\{\int_{E(0, t \rho(\lambda))}+\int_{\beta(0, y) \geq t \rho(\lambda)}\right\}\left|f \circ \varphi_{x}(y)-\hat{f}(x, t \rho(\lambda))\right|^{2} d v_{\lambda}(y) \\
& =: I_{1, \lambda, t}(x)+I_{2, \lambda, t}(x) .
\end{aligned}
$$

First we estimate the integral $I_{1, \lambda, t}(x)$ using the transformation rule together with the Cauchy-Schwarz inequality:

$$
\begin{aligned}
& I_{1, \lambda, t}(x)=\int_{E(x, t \rho(\lambda))}\left|k_{x}^{\lambda}(y)\right|^{2}|f(y)-\hat{f}(x, t \rho(\lambda))|^{2} d v_{\lambda}(y) \\
& \leq c_{\lambda}\left\{\int_{E(x, t \rho(\lambda))}\left|k_{x}^{\lambda}(y)\right|^{4}\left(1-|y|^{2}\right)^{2(\lambda-p)} d v(y)\right\}^{\frac{1}{2}}\left\{\int_{E(x, t \rho(\lambda))}|f(y)-\hat{f}(x, t \rho(\lambda))|^{4} d v(y)\right\}^{\frac{1}{2}} .
\end{aligned}
$$

We calculate the first integral on the right:

$$
\begin{aligned}
\int_{E(x, t \rho(\lambda))}\left|k_{x}^{\lambda}(y)\right|^{4} & \left(1-|y|^{2}\right)^{2(\lambda-p)} d v(y)= \\
& =\frac{1}{c_{2 \lambda-p}} \int_{E(x, t \rho(\lambda))}\left|k_{x}^{2 \lambda-p}(y)\right|^{2}\left|k_{x}^{p}(y)\right|^{2} d v_{2 \lambda-p}(y) \\
& =\frac{1}{c_{2 \lambda-p}} \int_{E(0, t \rho(\lambda))}\left|k_{x}^{p} \circ \varphi_{x}(y)\right|^{2} d v_{2 \lambda-p}(y)=(+) .
\end{aligned}
$$

According to Proposition 3 in [9], it holds

$$
\left|k_{x}^{p} \circ \varphi_{x}(y)\right|^{2}=\frac{1}{\left|k_{x}^{p}(y)\right|^{2}}=\frac{|h(y, x)|^{2 p}}{h(x, x)^{p}} .
$$

Therefore

$$
(+)=\frac{1}{c_{2 \lambda-p} h(x, x)^{p}} \int_{E(0, t \rho(\lambda))}|h(y, x)|^{2 p} d v_{2 \lambda-p}(y) \leq\|h\|_{\infty}^{2 p} \frac{|E(0, t \rho(\lambda))|}{h(x, x)^{p}},
$$

where $\|h\|_{\infty}=\sup _{x, y \in \mathbb{B}^{n}}|h(y, x)|<\infty$. According to Lemma 1.23 in [27], the volume of the Bergman ball $E(z, t \rho(\lambda))$ with $z \in \mathbb{B}^{n}$ is given by

$$
\begin{aligned}
|E(z, t \rho(\lambda))| & =\frac{\tanh (t \rho(\lambda))^{2 n}\left(1-|z|^{2}\right)^{n+1}}{\left(1-\tanh (t \rho(\lambda))^{2}|z|^{2}\right)^{n+1}} \\
& \leq C(t \rho(\lambda))^{2 n}\left(1-|z|^{2}\right)^{n+1}=\frac{t^{2 n} C}{c_{\lambda}} h(z, z)^{p} .
\end{aligned}
$$


Here $C>0$ is a suitable constant and the estimate holds for large $\lambda>n$. Inserting this estimate above with $z=0$ gives

$$
(+) \leq \frac{\|h\|_{\infty}^{2 p} t^{2 n} C}{c_{\lambda}} \frac{1}{h(x, x)^{p}}
$$

Hence we have

$$
I_{1, \lambda, t}(x) \leq\|h\|_{\infty}^{p} t^{n} \sqrt{C c_{\lambda}} \frac{|E(x, t \rho(\lambda))|^{\frac{1}{2}}}{h(x, x)^{\frac{p}{2}}}\left\{\frac{1}{|E(x, t \rho(\lambda))|} \int_{E(x, t \rho(\lambda))}|f(y)-\hat{f}(x, t \rho(\lambda))|^{4} d v(y)\right\}^{\frac{1}{2}} .
$$

By using (4.4) again one obtains as $\lambda \rightarrow \infty$ :

$$
I_{1, \lambda, t}(x) \leq\|h\|_{\infty}^{p} t^{2 n} C \sqrt{A_{4}(f, t \rho(\lambda), x)} \leq 2\|h\|_{\infty}^{p} t^{2 n} C\|f\|_{\infty} \sqrt{A_{2}(f, t \rho(\lambda), x)} \rightarrow 0,
$$

where by the assumption on $f$ the above limit is uniform on $\mathbb{B}^{n}$.

Now we estimate the second integral in (4.2) which we have denoted $I_{2, \lambda, t}(x)$. By [27, Corollary 1.22], we have $\beta(0, y) \geq t \rho(\lambda)$ if and only if $|y| \geq \tanh (t \rho(\lambda))=: R_{\lambda}$. A change of variables shows that

$$
\begin{aligned}
c_{\lambda} \int_{\beta(0, y) \geq t \rho(\lambda)}\left(1-|y|^{2}\right)^{\lambda-p} d v(y) & =2 n c_{\lambda} \int_{R_{\lambda}}^{1}\left(1-r^{2}\right)^{\lambda-n-1} r^{2 n-1} d r \\
& =2 n \frac{c_{\lambda}}{\lambda^{n}} t^{2 n} \int_{R_{\lambda} \frac{\sqrt{\lambda}}{t}}^{\frac{\sqrt{\lambda}}{t}}\left(1-\frac{t^{2} r^{2}}{\lambda}\right)^{\lambda-n-1} r^{2 n-1} d r=g\left(R_{\lambda}\right) .
\end{aligned}
$$

We have the following asymptotic behavior as $\lambda \rightarrow \infty$ :

$$
R_{\lambda}^{2}=\tanh (t \rho(\lambda))^{2} \sim \frac{t^{2}}{c_{\lambda}^{1 / n}} \sim \frac{t^{2}}{\lambda} \quad \text { and } \quad c_{\lambda} \sim \lambda^{n}
$$

Moreover, for all $\lambda>p-1$ and fixed $t>0$ the integrand is dominated by $c \exp \left(-t^{2} r^{2}\right) r^{2 n-1}$ where $c>0$ is a suitable constant independent of $t$ and $r$. Therefore the dominated convergence theorem implies:

$$
\lim _{\lambda \rightarrow \infty} g\left(R_{\lambda}\right)=2 n \gamma_{1} t^{2 n} \int_{\gamma_{2}}^{\infty} e^{-t^{2} r^{2}} r^{2 n-1} d r=: H(t)
$$

Since $H(t) \rightarrow 0$ as $t \rightarrow \infty$ we can choose $t>0$ sufficiently large such that for $\lambda>M_{1}$ and all $x \in \mathbb{B}^{n}$ we have

$$
I_{2, \lambda, t}(x) \leq 4\|f\|_{\infty}^{2} g\left(R_{\lambda}\right) \leq 8\|f\|_{\infty}^{2} H(t)<\varepsilon .
$$

With this fixed $t$ we can choose $M_{2}>M_{1}$ such that $I_{1, \lambda, t}(x) \leq \varepsilon$ for $\lambda>M_{2}$ and all $x \in \mathbb{B}^{n}$. From (4.2) we find $\mathrm{MO}^{\lambda}(f)(x)<2 \varepsilon$ uniformly on $\mathbb{B}^{n}$. 
Proposition 4.6. Let $\Omega$ be a BSD and $f \in \mathrm{BMO}^{\lambda}(\Omega)$. Then it holds

$$
\sup _{z \in \Omega} \mathcal{B}_{\lambda}\left(\left|f-\mathcal{B}_{\lambda}(f)\right|\right)(z) \leq C\|f\|_{\mathrm{BMO}^{\lambda}}
$$

for some constant $C>0$ (independent of $\lambda \geq 2 p$ ).

Proof. By the triangular inequality, we have for all $z \in \Omega$ :

$$
\begin{aligned}
\mathcal{B}_{\lambda}(\mid f & \left.-\mathcal{B}_{\lambda}(f) \mid\right)(z)=\int_{\Omega}\left|f(w)-\mathcal{B}_{\lambda}(f)(w)\right|\left|k_{z}^{\lambda}(w)\right|^{2} d v_{\lambda}(w) \\
& \leq \int_{\Omega}\left|f(w)-\mathcal{B}_{\lambda}(f)(z)\right|\left|k_{z}^{\lambda}(w)\right|^{2} d v_{\lambda}(w)+\int_{\Omega}\left|\mathcal{B}_{\lambda}(f)(z)-\mathcal{B}_{\lambda}(f)(w)\right|\left|k_{z}^{\lambda}(w)\right|^{2} d v_{\lambda}(w) .
\end{aligned}
$$

According to the Cauchy-Schwarz inequality the first term is dominated by $\|f\|_{\mathrm{BMO}^{\lambda}}$. Hence it remains to estimate the second term. Using [3, Theorem 4.9] and Corollary [3.3, we get

$$
\begin{aligned}
\int_{\Omega}\left|\mathcal{B}_{\lambda}(f)(z)-\mathcal{B}_{\lambda}(f)(w)\right|\left|k_{z}^{\lambda}(w)\right|^{2} d v_{\lambda}(w) \leq 2 \sqrt{\frac{\lambda}{p}}\|f\|_{\mathrm{BMO}^{\lambda}} \int_{\Omega} \beta(z, w)\left|k_{z}^{\lambda}(w)\right|^{2} d v_{\lambda}(w) \\
=2 \sqrt{\frac{\lambda}{p}}\|f\|_{\mathrm{BMO}^{\lambda}} \int_{\Omega} \beta\left(z, \varphi_{z}(w)\right) d v_{\lambda}(w) \\
=2 \sqrt{\frac{\lambda}{p}}\|f\|_{\mathrm{BMO}^{\lambda}} \int_{\Omega} \beta(0, w) d v_{\lambda}(w) \\
=\frac{2 C}{\sqrt{p}}\|f\|_{\mathrm{BMO}^{\lambda}} \int_{\Omega} h(w, w)^{-\frac{\lambda}{2}} d v_{\lambda}(w) \\
=\frac{2 C}{\sqrt{p}} \frac{c_{\lambda}}{c_{\frac{\lambda}{2}}}\|f\|_{\mathrm{BMO}^{\lambda}} .
\end{aligned}
$$

Here $C>0$ is the constant in Corollary 3.3 which does not depend on $\lambda$. Since the quotient $\frac{c_{\lambda}}{c_{\frac{\lambda}{2}}}$ is bounded as a function of $\lambda$, the proposition follows.

Lemma 4.7. Let $\Omega$ be a BSD and $f \in L^{\infty}(\Omega)$. Then there is a constant $C>0$ (independent of $\lambda \geq 2 p$ ) such that

$$
\left\|T_{f}^{\lambda}\right\|_{\lambda} \leq C \sqrt{\|f\|_{\infty}\left\|\mathcal{B}_{\frac{\lambda}{2}}(|f|)\right\|_{\infty}}
$$

where we consider $T_{f}^{\lambda}=P_{\lambda} M_{f}$ as an operator acting on the whole space $L^{2}\left(\Omega, d v_{\lambda}\right)$.

Proof. We use the Schur test and put $h_{\lambda}(z):=h(z, z)^{-\frac{\lambda}{2}}, C:=\sup _{\lambda \geq 2 p} \frac{c_{\lambda}}{c_{\frac{\lambda}{2}}}$. Recall that the Toeplitz operator $T_{f}^{\lambda}$ has the integral kernel

$$
T_{\lambda, f}(z, w):=f(z) h(z, w)^{-\lambda} .
$$


Hence we obtain

$$
\begin{aligned}
\int_{\Omega}\left|T_{\lambda, f}(z, w)\right| h_{\lambda}(z) d v_{\lambda}(z) & =c_{\lambda} \int_{\Omega}|f(z)||h(z, w)|^{-\lambda} h(z, z)^{\frac{\lambda}{2}-p} d v(z) \\
& =\frac{c_{\lambda}}{c_{\frac{\lambda}{2}}} \int_{\Omega}|f(z)||h(z, w)|^{-\frac{\lambda}{2} \cdot 2} d v_{\frac{\lambda}{2}}(z) \\
& =\frac{c_{\lambda}}{c_{\frac{\lambda}{2}}} h_{\lambda}(w) \mathcal{B}_{\frac{\lambda}{2}}(|f|)(w) \\
& \leq C_{1} h_{\lambda}(w)
\end{aligned}
$$

with $C_{1}=C\left\|\mathcal{B}_{\frac{\lambda}{2}}(|f|)\right\|_{\infty}$. Integration with respect to the parameter $w$ yields:

$$
\begin{aligned}
\int_{\Omega}\left|T_{\lambda, f}(z, w)\right| h_{\lambda}(w) d v_{\lambda}(w) & =c_{\lambda}|f(z)| \int_{\Omega}|h(z, w)|^{-\lambda} h(w, w)^{\frac{\lambda}{2}-p} d v(w) \\
& =\frac{c_{\lambda}}{c_{\frac{\lambda}{2}}}|f(z)| \int_{\Omega}|h(z, w)|^{-\frac{\lambda}{2} \cdot 2} d v_{\frac{\lambda}{2}}(w) \\
& =\frac{c_{\lambda}}{c_{\frac{\lambda}{2}}}|f(z)| h(z, z)^{-\frac{\lambda}{2}} \\
& \leq C_{2} h_{\lambda}(z)
\end{aligned}
$$

with $C_{2}=C\|f\|_{\infty}$. Hence $\left\|T_{f}^{\lambda}\right\|_{\lambda}$ is dominated by $\sqrt{C_{1} C_{2}}=C \sqrt{\|f\|_{\infty}\left\|\mathcal{B}_{\frac{\lambda}{2}}(|f|)\right\|_{\infty}}$.

Corollary 4.8. Let $\Omega$ be a $B S D, f \in L^{\infty}(\Omega)$ and $C>0$ as above. Then

$$
\left\|H_{f}^{\lambda}\right\|_{\lambda} \leq C \sqrt{\|f\|_{\infty}\left\|\mathcal{B}_{\frac{\lambda}{2}}(|f|)\right\|_{\infty}} .
$$

Proof. Note that the norm of the multiplication $M_{f}: \mathcal{A}_{\lambda}^{2}(\Omega) \rightarrow L^{2}\left(\Omega, d v_{\lambda}\right)$ coincides with the norm of

$$
M_{f} P_{\lambda}=\left[P_{\lambda} M_{\bar{f}}\right]^{*} \in \mathcal{L}\left(L^{2}\left(\Omega, d v_{\lambda}\right)\right)
$$

Therefore one has

$$
\left\|H_{f}^{\lambda}\right\|_{\lambda} \leq\left\|M_{f}: \mathcal{A}_{\lambda}^{2}(\Omega) \rightarrow L^{2}\left(\Omega, d v_{\lambda}\right)\right\|_{\lambda}=\left\|P_{\lambda} M_{\bar{f}}\right\|_{\lambda}
$$

and Lemma 4.7 implies the estimate (4.5).

Theorem 4.9. Let $f \in \operatorname{VMO}\left(\mathbb{B}^{n}\right)$. Then $\lim _{\lambda \rightarrow \infty}\left\|H_{f}^{\lambda}\right\|_{\lambda}=0$ and, in particular, we obtain

$$
\lim _{\lambda \rightarrow \infty}\left\|T_{f}^{\lambda} T_{g}^{\lambda}-T_{f g}^{\lambda}\right\|_{\lambda}=0
$$

for all $g \in L^{\infty}\left(\mathbb{B}^{n}\right)$ or all $g \in \mathrm{UC}\left(\mathbb{B}^{n}\right)$. 
Proof. Because of (2.10) and Theorem 3.8 (in the case where $g \in \mathrm{UC}\left(\mathbb{B}^{n}\right)$ ) it is sufficient to check that for all $f \in \operatorname{VMO}\left(\mathbb{B}^{n}\right)$ :

According to Corollary 4.8, we have:

$$
\lim _{\lambda \rightarrow \infty}\left\|H_{f}^{\lambda}\right\|_{\lambda}=0
$$

$$
\begin{aligned}
\left\|H_{f}^{\lambda}\right\|_{\lambda} & \leq\left\|H_{f-\mathcal{B}_{\frac{\lambda}{2}}(f)}^{\lambda}\right\|_{\lambda}+\left\|H_{\mathcal{B}_{\frac{\lambda}{2}}(f)}^{\lambda}\right\|_{\lambda} \\
& \leq C \sqrt{2\|f\|_{\infty}\left\|\mathcal{B}_{\frac{\lambda}{2}}\left(\left|f-\mathcal{B}_{\frac{\lambda}{2}}(f)\right|\right)\right\|_{\infty}}+\left\|H_{\mathcal{B}_{\frac{\lambda}{2}}(f)}^{\lambda}\right\|_{\lambda} .
\end{aligned}
$$

The first term on the right tends to zero as $\lambda \rightarrow \infty$ by the Proposition 4.6 and Theorem 4.5, Note that according to (2.5) we have $\sqrt{2} \beta_{\frac{\lambda}{2}}(z, w)=\beta_{\lambda}(z, w)$ and Theorem 3.5 implies that

$$
\left\|\mathcal{B}_{\frac{\lambda}{2}}(f)\right\|_{\mathrm{BO}^{\lambda}} \leq \sqrt{2}\|f\|_{\mathrm{BMO}^{\frac{\lambda}{2}}} .
$$

The estimate (3.6) implies that there is $C>0$ independent of $\lambda$ and $f$ with

$$
\left\|H_{\mathcal{B}_{\frac{\lambda}{2}}^{\lambda}(f)}^{\lambda}\right\|_{\lambda} \leq C\left\|\mathcal{B}_{\frac{\lambda}{2}}(f)\right\|_{\mathrm{BO}^{\lambda}} \leq \sqrt{2} C\|f\|_{\mathrm{BMO}^{\frac{\lambda}{2}}} .
$$

Since $f \in \operatorname{VMO}\left(\mathbb{B}^{n}\right)$ it follows from Theorem 4.5 that the right hand side tends to zero as $\lambda \rightarrow \infty$. Hence (4.7) proves the assertion.

We add an observation on the asymptotic behavior of semi-commutators of Toeplitz operator and a relation to a compactness result in [5]. With $n \in \mathbb{N}$ consider the standard monomial orthonormal basis of $\mathcal{A}_{\lambda}^{2}\left(\mathbb{B}^{n+1}\right)$

$$
\mathcal{B}=\left\{e_{\alpha}^{\lambda}(z):=z^{\alpha}\left\|z^{\alpha}\right\|_{\lambda}^{-1}: \alpha \in \mathbb{Z}_{+}^{n+1}\right\} .
$$

We split the coordinates $z \in \mathbb{B}^{n+1}$ and multi-indices $\alpha \in \mathbb{Z}_{+}^{n+1}$ into two parts:

$$
z=\left(z^{\prime}, z^{\prime \prime}\right) \in \mathbb{C} \times \mathbb{C}^{n} \quad \text { and } \quad \alpha=\left(\alpha^{\prime}, \alpha^{\prime \prime}\right) \in \mathbb{Z}_{+} \times \mathbb{Z}_{+}^{n} .
$$

A direct calculation (cf. [4] in the case of $n=1$ ) shows that $e_{\alpha}^{0}(z)=e_{\alpha^{\prime}}^{n}\left(z^{\prime}\right) e_{\alpha^{\prime \prime}}^{\left|\alpha^{\prime}\right|+1}\left(z^{\prime \prime}\right)$. This induces an orthogonal decomposition of the unweighted Bergman space

$$
\mathcal{A}_{0}^{2}\left(\mathbb{B}^{n+1}\right)=\bigoplus_{j \in \mathbb{Z}_{+}} \underbrace{\operatorname{span}\left\{e_{\alpha^{\prime}}^{n}\left(z^{\prime}\right): \alpha^{\prime}=j\right\} \otimes \mathcal{A}_{j+1}^{2}\left(\mathbb{B}^{n}\right)}_{=: H_{j}} .
$$

Let $c, d \in L^{\infty}\left(\mathbb{B}^{n}\right)$ and extend $c$ to the ball $\mathbb{B}^{n+1}$ by $f_{c}(z):=c\left(z^{\prime \prime}\right)$. The Toeplitz operator $T_{f_{c}}^{0}$ and the semi-commutator $T_{f_{c}}^{0} T_{f_{d}}^{0}-T_{f_{c} f_{d}}^{0}$ act on (4.8) as follows:

$$
\begin{gathered}
T_{f_{c}}^{0}=\bigoplus_{j=0}^{\infty} I \otimes T_{c}^{j+1} \\
T_{f_{c}}^{0} T_{f_{d}}^{0}-T_{f_{c} f_{d}}^{0}=\bigoplus_{j=0}^{\infty} I \otimes\left(T_{c}^{j+1} T_{d}^{j+1}-T_{c d}^{j+1}\right) .
\end{gathered}
$$


Assume that $f_{c}$ or $f_{d}$ are functions in $\mathrm{VMO}_{\partial}\left(\mathbb{B}^{n+1}\right)$. Then it follows from the results in [5] that the semi-commutator $T_{f_{c}}^{0} T_{f_{d}}^{0}-T_{f_{c} f_{d}}^{0}$ is compact on $\mathcal{A}_{0}^{2}\left(\mathbb{B}^{n+1}\right)$. The decomposition (4.9) and standard arguments imply that

$$
T_{c}^{j+1} T_{d}^{j+1}-T_{c d}^{j+1} \in \mathcal{K}\left(\mathcal{A}_{j+1}^{2}\left(\mathbb{B}^{n}\right)\right) \quad \text { and } \quad \lim _{j \rightarrow \infty}\left\|T_{c}^{j+1} T_{d}^{j+1}-T_{c d}^{j+1}\right\|_{j}=0 .
$$

Introduce the $C^{*}$-algebra

$$
\operatorname{VMO}^{\dagger}\left(\mathbb{B}^{n}\right):=\left\{c \in L^{\infty}\left(\mathbb{B}^{n}\right): f_{c} \in \mathrm{VMO}_{\partial}\left(\mathbb{B}^{n+1}\right)\right\} .
$$

Observe that if $z$ tends to $z_{0}=\left(z_{0}^{\prime}, z_{0}^{\prime \prime}\right) \in \partial \mathbb{B}^{n}$, with any $z_{0}^{\prime} \neq 0$, then the values of the function $f_{c}$ near the boundary point $z_{0}$ coincide with the values of the function $c$ in a neighborhood of the point $z_{0}^{\prime \prime} \in \mathbb{B}^{n}$. Hence a "vanishing oscillation condition" of the function $f_{c}$ near the boundary may be interpreted as a "vanishing oscillation condition" of the function $c$ inside $\mathbb{B}^{n}$. This somewhat vague comment suggests the following

conjecture: $\operatorname{VMO}^{\dagger}\left(\mathbb{B}^{n}\right)=\operatorname{VMO}\left(\mathbb{B}^{n}\right)$.

\section{REFERENCES}

[1] W. Bauer, L.A. Coburn, Uniformly continuous functions and quantization on the Fock space, Bol. Soc. Mat. Mex. (3) 22 (2016) no. 2, 669-677.

[2] W. Bauer, L.A. Coburn, Toeplitz operators with uniformly continuous symbols, Integr. Equ. Oper. Theory 83 (2015) no. 1, 25-34.

[3] W. Bauer, L.A. Coburn, Heat flow, weighted Bergman spaces and real analytic Lipschitz approximation, J. Reine Angew. Math. 703, (2015), 225-246.

[4] W. Bauer, N. Vasilevski, On algebras generated by Toeplitz operators and their representations, J. Funct. Anal. 272 (2017), no. 2, 705-737.

[5] D. Békollé, C. A. Berger, L.A. Coburn, K.H. Zhu, BMO in the Bergman metric on bounded symmetric domains, J. Funct. Anal. 93 (1990), 310-350.

[6] F. A. BerezIn, Quantization, Izv. Akad. Nauk SSSR Ser. Mat. 38 (1974), 1116-1175.

[7] F. A. Berezin, Quantization in complex symmetric spaces, Izv. Akad. Nauk SSSR Ser. Mat. 39 (1975), no. 2, 363-402.

[8] F. A. Berezin, General Concept of Quantization, Commun. Math. Phys., 40 (1975), 135-174.

[9] C.A. Berger, L.A. Coburn, K.H. Zhu, Function theory on Cartan domains and the BerezinToeplitz symbol calculus, Amer. J. Math. 110 (1988) no. 5, 921-953.

[10] M. Bordemann, E. Meinrenken, M. Schlichenmaier, Toeplitz quantization of Kähler manifolds and $\mathfrak{g l}(n), n \rightarrow \infty$ limits, Comm. Math. Phys. 165 (1994) no. 2, 281-296.

[11] D. BorTHWick, Microlocal techniques for semi-classical problems in geometric quantization, In: Perspectives on Quantization. Contemp. Mathematics, vol. 214, AMS, Providence, RI, (1998), 23-37.

[12] D. Borthwick, A. Lesniewski, H. Upmeier, Non-perturbative deformation quantization of Cartan domains, J. Funct. Anal. 113 (1993) no. 1, 153-176.

[13] E. CaRtan, Sur les domaines bornés homogènes de l'espace de $n$ variables complexes, Abh. Math. Sem. Univ. Hamburg 11 (1935), 116-162.

[14] L.A. Coburn, Deformation estimates for Berezin-Toeplitz quantization, Comm. Math. Phys. 149 (1992) no. 2, 415-424. 
[15] M. Engliš, Weighted Bergman kernels and quantization, Comm. Math. Phys. 227 (2002) no.2, 211241.

[16] M. EngLiš, Compact Toeplitz operators via the Berezin transform on bounded symmetric domains, Integr. Equ. Oper. Theory 33 (1999) no. 4, 426-455.

[17] J. Faraut, A. KoránYi, Function spaces and reproducing kernels on bounded symmetric domains, J. Funct. Anal. 88 (1990), no. 1, 64-89.

[18] J. B. Garnett, D. E. Marshall, Harmonic Measure, Cambridge University Press, 2005.

[19] S. Grudsky, A. Karapetyants, N. Vasilevski, Toeplitz operators on the unit ball in $\mathbb{C}^{n}$ with radial symbols, J. Operator Theory 49 (2003) no. 2, 325-346.

[20] S. Klimek, A. Lesniewski, Quantum Riemann surface I. The unit disc, Comm. Math. Phys. 146 (1992) no.1, 103-122.

[21] X. Xiaonan, G. Marinescu, Berezin-Toeplitz quantization on Kähler manifolds, J. Reine Angew. Math. 662 (2012), 1-56.

[22] M. Rieffel, Deformation quantization of Heisenberg manifolds, Comm. Math. Phys. 122 (1989) no. 4, 531-562.

[23] W. Rudin, Function theory in the unit ball of $\mathbb{C}^{n}$, Springer 2008.

[24] R. M. Timoney, Bloch functions in several complex variables. I., Bull. London Math. Soc. 12 (1980) no. 4, 241-267; II, J. Reine Angew. Math. 319 (1980), 1-22.

[25] N. Vasilevski. On Toeplitz operators with piecewise continuous symbols on the Bergman space, Operator Theory: Advances and Applications, v. 170 (2007), 229-248.

[26] K. ZHU, Operator theory in function spaces, Math. Surveys and Monographs 138, AMS, Providence, RI, 2007.

[27] K. ZHU, Spaces of holomorphic functions in the unit ball, Graduate Texts Math. 226, Springer, 2005.

[28] K. Zhu, VMO, ESV and Toeplitz operators on the Bergman space, Trans. Amer. Math. Soc. 302 (1987) no. 2, 617-646.

INSTITUT FÜR ANALYSIS

Welfengarten 1, 30167 Hannover, Germany

E-mail address: bauer@math.uni-hannover.de

INSTITUT FÜR ANALYSIS

Welfengarten 1, 30167 Hannover, Germany

E-mail address: raffael.hagger@math.uni-hannover.de

Departamento de Matemáticas, CinVESTAV

Apartado Postal 14-740, 07000, México, D.F., México

E-mail address: nvasilev@math.cinvestav.mx 